\title{
Bending Moment and Cross-Section Deformation of a Box Profile
}

\author{
Stanisław Kut ${ }^{1 *}$, Feliks Stachowicz ${ }^{1}$ \\ 1 Rzeszow University of Technology, al. Powstańców Warszawy 12, 35-959 Rzeszów, Poland \\ * Corresponding author"s e-mail: stan_kut@prz.edu.pl
}

\begin{abstract}
In the process of plastic bending of thin-walled profiles, there is a significant deformation of the cross-section, which has a very significant impact on the course and effects of the shaping process construction products. In this paper, the experimental, analytical as well as numerical analyses of the box profile bending process enabled to establish the relationships determining the dependence of the cross-sectional form and bending moment on the bending curvature. The following paper discusses pure bending moment and the cross-sectional deformation of $21.5 \times 21.5 \times 1.8 \mathrm{~mm}$ and $25 \times 25 \times 2.5 \mathrm{~mm}$ square tubes made of the 6060 aluminium alloy. Satisfactory agreement of the experimental results and numerical calculations was obtained for the values of horizontal and vertical wall deflection, as well as for the experimental, calculated and numerical bending moment characteristics.
\end{abstract}

Keywords: stretch-bending, box profile, aluminium alloy, deformation, FEM

\section{INTRODUCTION}

Rectangular tubes are widely used as structural elements in numerous applications, such as automobiles, trains, vehicles, aircraft, and offshore structures. In the elastoplastic bending of thin-walled profiles, the cross-sections are subjected to deformations, which strongly influence the value of bending moment, the minimum bending radius and the amount of spring-back [1-6]. In the case of bending circular cross-section tubes, the parameter describing the degree of cross-sectional deformation is the degree of ovalization. In turn, for the tubes of square or rectangular cross-section (box profiles), it is not possible to determine a similar unique parameter because of the complex shape of the crosssection after bending. The research works on the plastic bending process of box profiles focus on describing the changes in the cross-sectional geometry and determining the impact of these changes on the value of the number of parameters characterizing the bending process have been and still are the subject of many research works. The subject of numerous publications involves both experimental research [2, 7] and theoretical considerations based on the deformation theory of plasticity [8-11], numerical modelling using FEM [3, 4, 12-15], as well as artificial intelligence identification [16].

The observations and measurements of the bent cross-section shape of the box-section have enabled to establish the relationships determining the dependence of the cross-sectional form on the bending curvature [2]. The following indices are assumed for description of the shape of a crosssection after bending (Fig. 1):

- $\mathrm{U}_{\mathrm{zw}}$ - deflection of the inner horizontal wall,

- $\mathrm{U}_{\mathrm{zz}}-$ deflection of the outer horizontal wall,

- $\mathrm{U}_{\mathrm{yw}}$ - deflection of the inner profile corners,

- $\mathrm{U}_{\mathrm{yz}}$ - deflection of the outer profile corners,

- $\mathrm{U}_{\mathrm{ym}}$ - maximum lateral displacement of the points of the wall beyond the original cross-section.

The horizontal walls were those that are normal to the bending plane, the vertical walls are those parallel to the bending plane - inner and outer refer to towards, and away, to the bending axis, respectively. On the basis of the preliminary results of experimental investigations it was found that the wall shape of the deformed cross-section 
can be described most conveniently by the functions [2]:

$$
U_{z}=\eta e^{-y^{2} / B^{2}}
$$

for horizontal walls,

$$
U_{y}=\alpha z^{2}+\beta z
$$

for vertical walls.

The analysis of the results of horizontal wall deflection revealed its dependence on the geometrical characteristics of the box profile and the square radius of curvature:

$$
\text { for } y=0 \rightarrow U_{z}=\eta=c \frac{B^{3} H}{t} \frac{1}{R^{2}}
$$

The values of constants $c$ determined by statistical analysis are: $\mathrm{c}_{\mathrm{w}}=1.57$ for the inner horizontal wall and $c_{z}=2.25$ for the outer horizontal wall.

The purpose of this study was mainly to verify the correctness of the numerical model used to determine the changes in the cross-sectional geometry of box profiles and the relationship between the bending moment and the bending curvature.

\section{Material and experimental procedure}

The study was carried out on the box profiles of $21.5 \times 21.5 \times 1.8 \mathrm{~mm}$ and $25 \times 25 \times 2.5 \mathrm{~mm}$ extruded from the 6060 aluminium alloy (chemical composition in \%: $0.53 \mathrm{Si} ; 0.48 \mathrm{Mg} ; 0.26 \mathrm{Fe} ; 0.12 \mathrm{Zn}$; $0.10 \mathrm{Mn} ; 0.09 \mathrm{Ti} ; 0.08 \mathrm{Cu} ; 0.04 \mathrm{Cr}$ - the remainder is Al). This type of material was chosen because the box profiles made of it have highly accurate cross-section shapes, which was considered to be an essential property for the validity of the measured results. The samples prepared from profile wall were tested for tension and the Hollomon strain-stress relationship in the form of $\sigma=322 \varepsilon^{0.22}$ showed very significant reproducibility. Other tensile parameters were as follows: yield stress $\mathrm{Rp}_{0.2}=61 \mathrm{MPa}$; ultimate strength $\mathrm{R}_{\mathrm{m}}=128 \mathrm{MPa}$ and Young modulus $\mathrm{E}=71 \mathrm{GPa}$.

The bending tests were carried out with the help of a self-developed device mounted on a testing machine (Fig. 2). The bending on four supports ensured that the pipes are loaded with a pure bending moment, without the use of lateral forces. During bending, the bending force was measured continuously (then the bending moment value was calculated). The bending tests were carried out until the phenomenon of deformation localization occurred in the form of local refraction of the profile (usually in the middle of the bent section of the tube). For several selected bending stages, the curvature of the profile was determined and the measurements of displacement of characteristic points of individual walls were performed to determine the shape of the cross-section and the components of the deformation state.

\section{Components of the strain state}

When bending box profiles, the way of moving individual points on the perimeter of the profile varies, which makes it necessary to analyse the state of deformation separately in horizontal and vertical walls. The following assumptions were made for the determination of the strain state components:

- the neutral axis lies in the central area of the cross-section, because of the application of only relatively small curves,

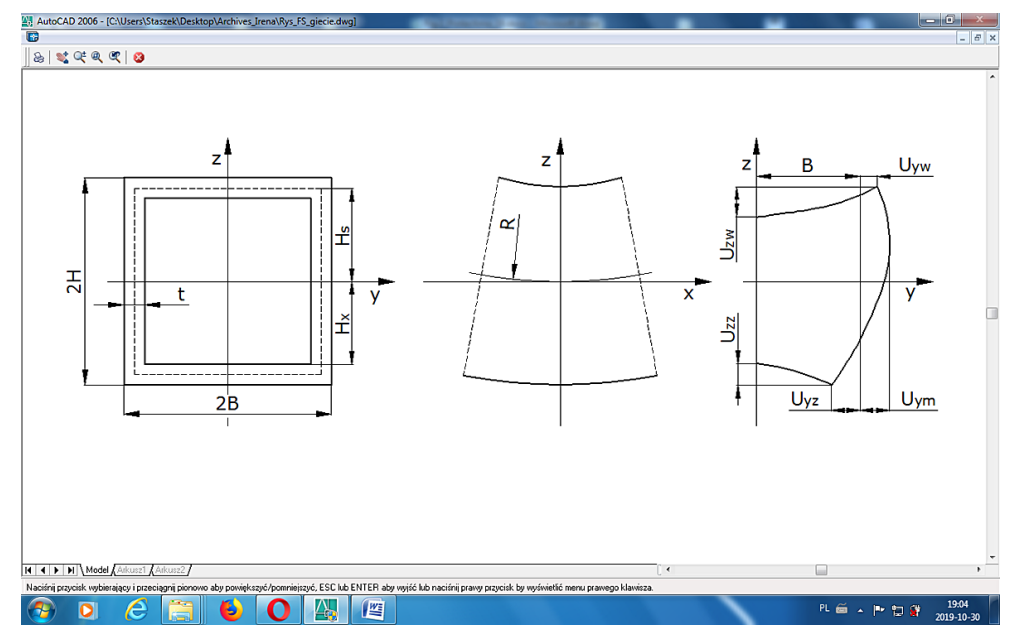

Fig. 1. Characteristic tube dimensions and displacements of the deformed cross section 


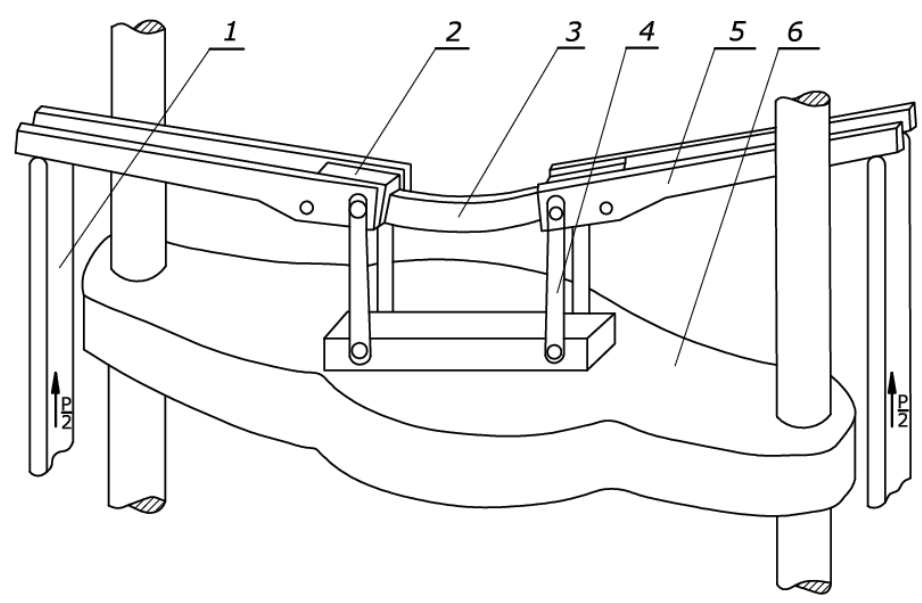

Fig. 2. Laboratory bending device with pure bending moment: 1 - lever, 2 - clamping jaws, 3 - sample, 4 - connector, 5 - bending arm, 6 - traverse of testing machine

- the postulation of plane cross-section is retained in the bending process, thus the strain state was determined for the central line of the cross-section,

- the mechanical characteristics of the material in the compression (inner) and tension (outer) zones are identical.

- According to the assumed scheme of horizontal wall displacement (Fig. 1), the individual components of the strain state have the forms: for the horizontal inner wall:

$$
\begin{aligned}
& \varepsilon_{x w}=\frac{H_{s}-u_{z w}(y)}{R} ; \varepsilon_{y w}= \\
= & a_{w} ; \varepsilon_{z w}=\varepsilon_{x w}-a_{w}
\end{aligned}
$$

for the horizontal outer wall:

$$
\begin{aligned}
& \varepsilon_{x z}=\frac{H_{s}-u_{z z}(y)}{R} ; \varepsilon_{y z}= \\
= & -a_{z} ; \varepsilon_{z z}=\varepsilon_{x z}-a_{z}
\end{aligned}
$$

and the value of effective strain:

$$
\varepsilon_{e}=\frac{2}{\sqrt{3}}\left(\sqrt{\varepsilon_{x}^{2}-a \varepsilon_{x}+a^{2}}\right.
$$

A constant value of deformation in the direction of the $y$ axis $(a=$ const) was assumed in order to simplify the calculations. By means of variation calculus [17] based on the Ritz method the value of parameter a can be determined from the condition of total minimum energy of the system:

$$
\partial E=\partial(W-A)
$$

The work of external forces is determined by the relationship:

$$
A=M \gamma
$$

where: $\boldsymbol{M}$ is a bending moment and $\boldsymbol{\gamma}$ is the rotation angle of the outer fibres of the cross-section.

And the work of internal forces dependence is:

$$
W=\iiint_{V}\left\{\int_{0}^{\varepsilon_{\theta}} \sigma d \varepsilon\right\} d V
$$

After using equations (1) and (4), the value of internal forces for horizontal walls will be:

$$
\begin{aligned}
& W_{\text {hor }}=\frac{2 K t B}{n+1}\left(\frac{2 H_{s}}{\sqrt{3} R}\right)^{n+1}\left\{1+\frac{n+1}{2}\right. \\
& \left(-1.5 \frac{\eta}{H_{s}}+0.6 \frac{\eta^{2}}{H_{s}^{2}}-\frac{a R}{H_{s}}+\frac{a^{2} R^{2}}{H_{s}^{2}}+0.75 \frac{a \eta R}{H_{s}^{2}}\right) \\
& \left.+\frac{n^{2}-1}{8}\left(\begin{array}{c}
2.4 \frac{\eta^{2}}{H_{s}^{2}}+\frac{a^{2} R^{2}}{H_{s}^{2}}+\frac{a^{4} R^{4}}{H_{s}^{4}}-\frac{2 a^{3} R^{3}}{H_{s}^{3}}- \\
-3.6 \frac{a \eta^{2} R}{H_{s}^{3}}+\frac{a \eta R}{H_{s}^{2}}-4.5 \frac{a^{2} \eta R^{2}}{H_{s}^{3}}-2.2 \frac{\eta^{3}}{H_{s}^{3}}+ \\
+1.2 \frac{a^{2} \eta^{2} R^{2}}{H_{s}^{4}}+1.5 \frac{a^{3} \eta R^{3}}{H_{s}^{4}}
\end{array}\right)\right\}
\end{aligned}
$$

Because in the expression for the work of external forces (6) the size of bending angle $\gamma$ does not depend on the parameter $\boldsymbol{a}$, the value of this parameter can be determined with the relation:

$$
\frac{d W}{d \gamma}=0
$$

After carrying out the prescribed calculations, the expression for the value of the parameter $\boldsymbol{a}$ takes the form:

$$
\mathrm{a}=\frac{\frac{n+1}{2}\left(11-0.75 \eta H_{S}\right)-\frac{3}{8}\left(n^{2}-1\right)\left(\frac{\eta}{H_{S}}\right)\left(1-1.5 \frac{\eta}{H_{S}}\right.}{(n+1) \frac{R}{H_{S}}+0.25\left(n^{2}-1\right)\left(1-4.5 \frac{\eta}{H_{S}}\right) \frac{R}{H_{S}}}
$$


In the case of vertical wall displacement (Fig. 1) considering equation (2), the individual components of the strain state have the forms:

$$
\begin{aligned}
& \varepsilon_{x}=-\frac{z}{R} ; \varepsilon_{y}=-\varepsilon_{x}-\varepsilon_{z} ; \\
& \varepsilon_{z}=\sqrt{1+(\alpha z+\beta)^{2}-1}
\end{aligned}
$$

The magnitudes of $\boldsymbol{\alpha}$ and $\boldsymbol{\beta}$ were determined from the condition relating the displacements of the horizontal and vertical walls in the profile corners:

$$
\begin{aligned}
U_{y w} & =\sqrt{\left(1+a_{w}\right)^{2} B^{2}-\eta_{w}^{2}}-B \\
U_{y z} & =\sqrt{\left(1+a_{z}\right)^{2} B^{2}-\eta_{z}^{2}}-B
\end{aligned}
$$

The solution of the equation system presented yields:

$$
\alpha=\frac{U_{y w}+U_{y z}}{2 H^{2}} \text { and } \beta=\frac{U_{y w}-U_{y z}}{2 H}
$$

The maximum displacement of the vertical wall determined as the extremum of the assumed parabola (2) has the value:

$$
U_{y m}=-\frac{\beta^{2}}{4 \alpha}=\frac{\left(U_{y w}-U_{y z}\right)^{2}}{8\left(U_{y w}+U_{y z}\right)}
$$

\section{Bending moment}

The variable parameter that determines the size of the bending moment in the bending process is the value of curvature $\kappa=1 / R$. Therefore, we differentiate dependence (6) with respect to the curvature and yields:

$$
M=\frac{\partial W}{\partial\left(\frac{1}{R}\right)}
$$

In order to determine the value of the bending moment of horizontal walls, we differentiate with respect to the curvature of the equation for the work of internal forces (9) for these walls and we obtain:

$$
\begin{gathered}
M_{h o r}=\frac{2 K t B}{n+1}\left(\frac{2 H_{s}}{\sqrt{3}}\right)^{n+1} \\
R^{-1}\left(1+n+\frac{n+1}{2} \lambda+\frac{n^{2}-1}{8} \lambda^{2}\right.
\end{gathered}
$$

where:

$$
\begin{gathered}
\lambda=-\frac{1.5 \eta(n+1)}{H_{s}}+\frac{0.6 \eta^{2}(n+1)}{H_{s}^{2}}- \\
-\frac{n a R}{H_{S}}+\frac{0.75 n a \eta R}{H_{S}^{2}}+\frac{(n-1) a^{2} R^{2}}{H_{s}^{2}}
\end{gathered}
$$

The calculations should be carried out separately for the inner wall $\left(a_{w}\right.$ and $\left.\eta_{w}\right)$ and the outer wall $\left(a_{z}\right.$ and $\left.\eta_{z}\right)$.

After using equations (8) and (12), the expression of internal forces for vertical walls takes the form:

$$
W_{\text {ver }}=\frac{2 K}{n+1}\left(\frac{2}{\sqrt{3}}\right)^{n+1} \int_{-\frac{t}{2}}^{\frac{t}{2}} \int_{0}^{H_{x}} \varepsilon_{e}^{n+1} d y d z
$$

After performing the prescribed calculations, the expression for calculating the bending moment value for horizontal walls will take the form:

$$
M_{\text {ver }}=\frac{2 K t}{n+1}\left(\frac{2}{\sqrt{3}}\right)^{n+1} R^{-n} H_{x}^{n+2}
$$

where: $H_{x}=H-t$

The $\mathrm{H}_{\mathrm{x}}$ value was introduced to avoid double bending moment calculation for profile corners.

The total bending moment of the box profile will be the sum of the bending moments for the horizontal (internal and external) walls and vertical walls:

$$
M_{\Sigma}=M_{h o r}^{Z}+M_{h o r}^{w}+2 M_{v e r}
$$

\section{Numerical calculations}

FEM modelling of the bending process was performed for two types of numerical models. The first is a full 3D model analysed in a spatial state of stress, while the other is a shell type model. Both numerical models of the bending process were built on the basis of an experiment, maintaining the same geometrical parameters of both the bending beam and the bending device. Due to the occurrence during bending of the tested beam, two planar symmetries (along the beam axis and perpendicular to the cross-section in the middle between the supports) numerical models were simplified to $1 / 4$ parts of the experimental model. Numerical modelling was carried out using a commercial MSC system, MARC/Mentat, which is considered as one of the most advanced programs for the MES analysis [18], in particular for the modelling of highly non-linear and contact issues. In both models, the beam was defined as a deformable body, while the elements of the device being in contact with it as perfectly rigid bodies.

In order to discretize the deformable body in the 3D model, 8-node hex-shaped elements type 7 [19] were used. The size of the elements on the cross-section of the beam was $0.625 \times 0.625 \mathrm{~mm}$, 
while on its length it amounted to $1 \mathrm{~mm}$, which gave a total of 54,720 finite elements. In order to discretize the deformable beam in the shell model (shell), 4-node quad 4 bilinear elements of type 75 [19] were used with the formula considering the effects of transverse shear [20], improving the behaviour of the shell elements during bending. The use of this type of mesh in coating models (e.g. pressing processes) enables to obtain good accuracy of calculations, with simultaneous savings in the form of shortening the calculation time [21]. The height of elements on the cross-section of the beam was $0.625 \mathrm{~mm}$ and their width on the beam length was $1 \mathrm{~mm}$, which gave a total of 13,680 elements.

The other simulations, the parameters for both numerical models were the same. The elastic-plastic model of the beam material was adopted with the non-linear strengthening described by the Hollomon power equation. The isotropic properties of the beam material were assumed both in the area of elastic and plastic deformation. The values of material constants in terms of elastic deformation of the material were: Young's modulus of elasticity E $=71.0 \mathrm{GPa}$, Poisson's ratio $v=0.33$. The curve of strain hardening determined on the basis of the uniaxial tensile test was introduced into the program in the tabular form. The evolution of the surface of plasticity as a result of the phenomenon of strain hardening was described using the isotropic model. The HuberMises-Hencky plasticity condition, the associated law of plastic flow of Prandtl-Russ and the implicit scheme of integration of differential equations over time with the Newton-Raphson method were used in the calculations.

\section{RESULTS AND DISCUSSION}

The results of numerical calculations allow noticing that the distortions of the equivalent deformations on the section of the $25 \times 25 \times 2.5 \mathrm{~mm}$ profile sample subjected to the bending moment, obtained for the 3D model (Fig. 3) are slightly different from those obtained for the shell model (Fig. 4). The strain values in the extreme fibres of the bent profile are higher for the 3D model than for the shell model. It should be noted here that the calculation time for the 3D Model (87673 s) was 8 times longer than for the shell type (11065 s). The results of numerical calculations presented in Figure 5 confirm the assumption that the neutral axis lies in the central area of the cross-section only for relatively small curves. The central position of the neutral plane can be assumed for bending curvature $\kappa \leq 4.0 \mathrm{~m}^{-1}$ (Fig. $5 \mathrm{a}$ ); for larger bending curvatures, the position of the neutral plane (Fig. 5b and 5c) shifts towards the bending axis (internal horizontal wall of the bent profile).

The numerical calculations of the displacement values of selected points on the $25 \times 25 \times 2.5$ $\mathrm{mm}$ profile cross-section occurring with the increase in curvature were compared with the results of measurements carried out during the experimental studies. In the case of the changes in the degree of the deflection of horizontal walls, both internal and external, good compliance of the results of calculations and the experiment was obtained (Fig. 6). Higher values of wall deflection, and also closer to the results of the experiment, were obtained using the shell method. It is worth noting that for the value of bending curvature equal to $0<\kappa<1 \mathrm{~m}^{-1}$, the deflection of the horizontal inner wall has the opposite direction than at a later stage of the bending process - this phenomenon was observed earlier [2, 22] for the elastic bending phase. Larger differences between the results of numerical calculations and the results of the experiment found in the case of the analysis of the degree of vertical wall bending (Fig. 7) - this time closer to the results of the experiment, were obtained using 3D calculation. It should be noted that the bending of the vertical walls of the profile cross-section slightly affects the value of the moment of the cross-section inertia, which determines the value of the bending moment.

On the basis of a comparison of the experimental tests results, the dependence of the bending moment on the value of the bending curvature and the results of the analytical and numerical calculations of this parameter (Fig. 8 and Fig. 9), it is possible to conclude that a better agreement of the characteristics was obtained for a profile with the dimensions of $21.5 \times 21.5 \times 1.8 \mathrm{~mm}$ than for a profile with the dimensions of $25 \times 25 \times 2.5 \mathrm{~mm}$.

In the case of a profile with larger crosssectional dimensions, very good consistency between the rigidity and the experimental bending characteristics can be seen for the analytically determined curve (Fig. 8). The course of bending characteristics determined numerically (both with the shell method and 3D method) differs from the course of the characteristic determined experimentally in the range of bending curvatures $\kappa>5 \mathrm{~m}^{-1}$. 


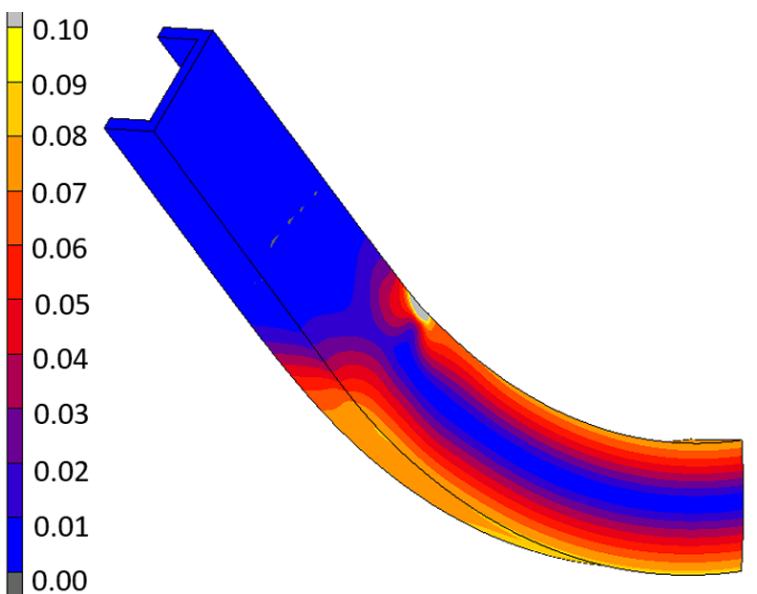

Fig. 3. An exemplary distribution of equivalent plastic deformations obtained from the 3D model $\left(\kappa=7 \mathrm{~m}^{-1}\right)$

For a profile with larger cross-section dimensions of $21.5 \times 21.5 \times 1.8 \mathrm{~mm}$, the best agreement of the experimental bending characteristics can be seen for the curve determined numerically with the shell method (Fig. 9). The courses of the characteristics determined analytically and numerically with the 3D method are very similar and differ from the characteristic determined experimentally in the range of curvatures $\kappa>6 \mathrm{~m}^{-1}$.

When analysing the course of bending characteristics, the phenomenon of the bending moment value in the final phase of the process for bending curvatures $\kappa>6.0$ was considered. A clear decrease in the bending moment value does not correspond to the monotonic increase in the degree of bending of the individual walls of the cross-section of the profile (Fig. 6 and Fig. 7). Therefore, it

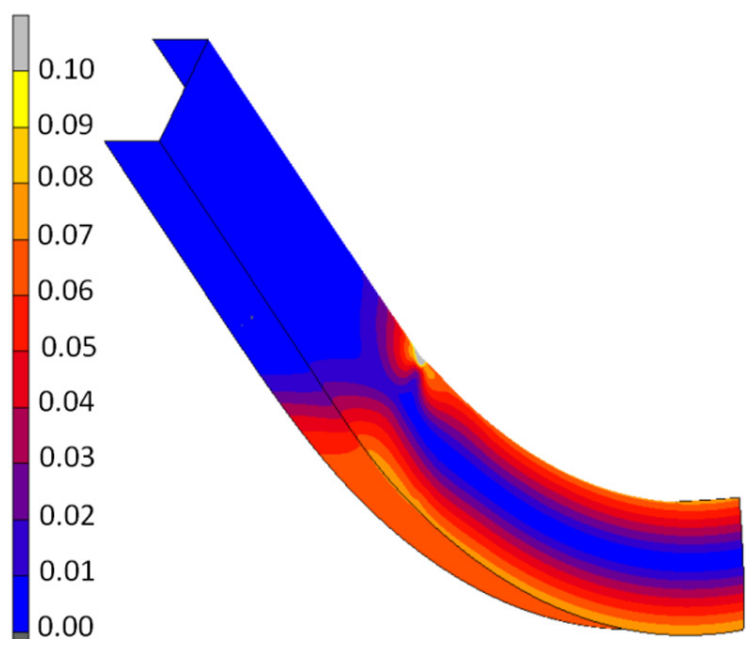

Fig. 4. An exemplary distribution of equivalent plastic deformations obtained from the shell model $\left(\kappa=7 \mathrm{~m}^{-1}\right)$

can be explained that during the experiment, due to the design of the bending device, the bending arm increased, which resulted in a decrease in the bending force, and consequently a reduction in the value of the calculated bending moment. For the bending curvatures $\kappa>9.0 \mathrm{~m}^{-1}$, a clearly increased wall deflection was observed, which consequently led to the local collapse of the pipe, and for these values of bending curvature, a decrease in the bending moment value is expected.

\section{CONCLUSION}

On the basis of the obtained results, it can be concluded that the developed numerical

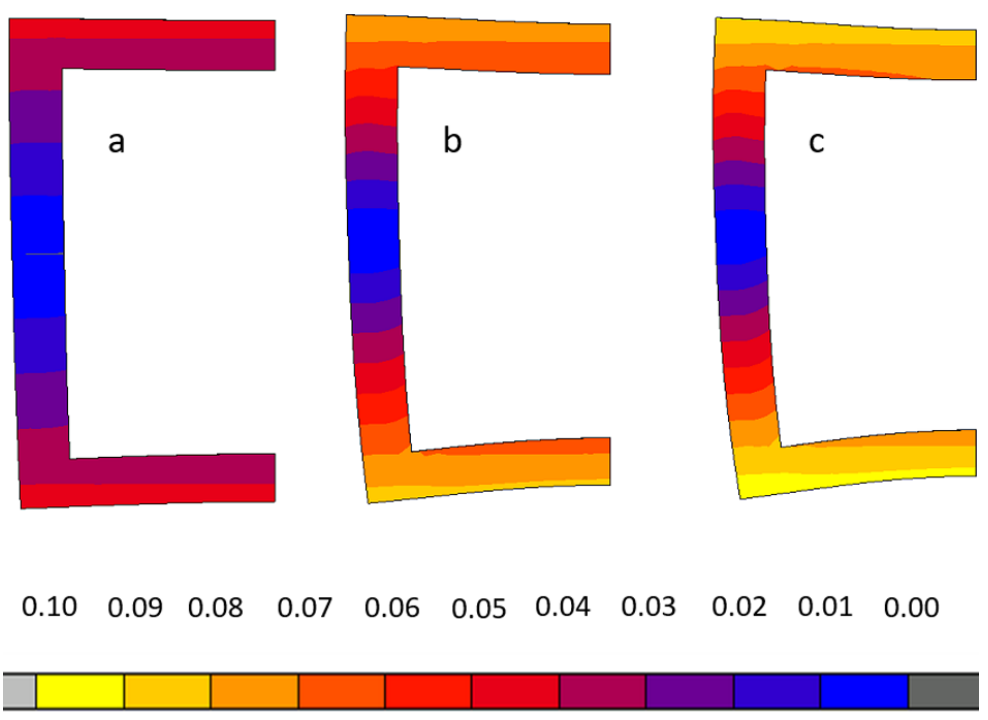

Fig. 5. Changing the shape of the cross-section of a bent beam (3D model) and distribution of equivalent plastic deformations for different bending curvature: a) $\kappa=4 \mathrm{~m}^{-1}$, b) $\kappa=7 \mathrm{~m}^{-1}$, c) $\kappa=8 \mathrm{~m}^{-1}$ 




Fig. 6. The effect of bending curvature on the deflection of horizontal walls of the $25 \times 25 \times 2.5 \mathrm{~mm}$ profile



Fig. 7. The effect of bending curvature on the deflection of vertical wall of the $25 \times 25 \times 2.5 \mathrm{~mm}$ profile



Fig. 8. The effect of bending curvature on the bending moment of the $25 \times 25 \times 2.5 \mathrm{~mm}$ profile 




Fig. 9. The effect of bending curvature on the bending moment of the $21.5 \times 21.5 \times 1.8 \mathrm{~mm}$ profile

calculations, both for the shell and the 3D model, describe well the analyzed parameters of the box profile bending process, i.e. the changes in crosssectional geometry and the value of the bending moment as a function of bending curvature. Satisfactory agreement of the experimental results and numerical calculations was obtained for the values of the horizontal wall deflection, while for the vertical walls it is significantly smaller, which does not significantly affect the results of calculations of the bending moment value for the bending curvature $\kappa<6 \mathrm{~m}^{-1}$, i.e. to the beginning phase of the deformation location.

\section{REFERENCES}

1. Kecman D. Bending collapse of rectangular and square section tubes. International Journal of Mechanical Science, 25, 1983, 623-636.

2. Świątkowski K. and Stachowicz F. Analysis of the cross-sectional deformation of box profiles subjected to bending. Journal of Mechanical Working Technology, 10, 1984, 103-116.

3. Chen D.H. and Masuda K. Rectangular hollow section in bending: Part I - Cross-sectional flattening deformation. Thin-Walled Structures, 106, 2016, 495-507.

4. Shen J. and Wadee M.A. Sensitivity to local imperfections in inelastic thin-walled rectangular hollow section struts. Structures, 17, 2019, 43-57.

5. Paulsen F., Welo T. and Sovik O.P. A design method for rectangular hollow sections in bending. Journal of Materials Processing Technology, 113, 2001, 699-704.
6. Zhu H. and Stelson K.A. Distortion of rectangular tubes in stretch bending. Journal of Manufacturing Science and Engineering, 124, 2002, 886-890.

7. Miller J.E., Kyriakides S. and Bastard A.H. On bend-stretch forming of aluminum extruded tubes - I: experiments, International Journal of Mechanical Science, 43, 2001, 1283-1317.

8. Corona E. and Vaze S. Buckling of elastic-plastic square tubes under bending. International Journal of Mechanical Science, 38, 1996, 753-775.

9. T.H. Kim and S.R. Reid: Bending collapse of thinwalled rectangular section columns, Computers \& Structures, 79, 2001, 1897-1911.

10. Paulsen F. and Welo T. A design method for prediction of dimensions of rectangular hollow sections formed in stretch bending. Journal of Materials Processing Technology, 128, 2002, 48-66.

11. Petrone F. and Monti G.: Unified code-compliant equations for bending and ductility capacity of full and hollow rectangular RC sections. Engineering Structures, 141, 2019, 805-815.

12. Chiew S.P., Jin Y.F. and Lee C.K.: Residual stress distribution of roller bending of steel rectangular structural hollow sections. Journal of Constructional Steel Research, 119, 2016, 85-97.

13. Shim D-S., Kim, K-P. and Lee K-Y.: Double-stage forming using critical pre-bending radius in roll bending of pipe with rectangular cross-section. Journal of Materials Processing Technology, 236, 2016, 189-203.

14. Lan X., Chen J., Chan T-K. and Young B. The continuous strength method for the design of high strength steel tubular sections in bending, Journal of Constructional Steel Research, 160, 2019, 499-509.

15. Zhu H. and Stelson K.A. Modeling and closed-loop 
control of stretch bending of aluminum rectangular tubes. Journal of Manufacturing Science and Engineering, 125, 2003, 113-119.

16. Litwin P. and Stachowicz F. Artificial intelligence identification of box profile bending parameters, Journal of Artificial Intelligence Study, 1, 2004, 99-106.

17. Zeidler E. Variational problems, the Ritz method, and the idea of orthogonality. in: Nonlinear functional analysis and its application, Springer, New York 1990.

18. Rojek J. Modelowanie i symulacja komputerowa złożonych zagadnień mechaniki nieliniowej meto- dami elementów skończonych i dyskretnych, Prace IPPT PAN, 4/2007, Warszawa 2007.

19. MSC Software, MSC.Marc Volume B, Element Library.

20. MSC Software, MSC.Marc Volume A, Theory and User Information.

21. Żmudzki A., Śledzińska A., Pietrzyk M., Woźnika H., Plewiński A., and Drenger T. Kontrola jakości produktów głębokiego tłoczenia blach za pomocą symulacji komputerowych. Obróbka Plastyczna, $16,2005,50-62$.

22. Oehler G. Querschnittsänderung an gebogenen profilen. Konstruction, 26, 1974, 171-176. 